\title{
A spherically topological analysis of stationary black holes
}

\author{
Benjamin Puzantian
}

\begin{abstract}
A black hole with zero angular momentum is said to be stationary and under certain conditions such a black hole can represented as a sphere. This review examines Hawking's topology theorem, the Schwarzschild metric, novel solutions to Einstein's equations, resonances of hyperbolic orbits around the event horizon for spherical, stationary black holes, and analyzes their importance. It is suggested, that in the spherical stationary black hole case, the Fourier analysis can be used to find the resonances due to Geometric scattering of hyperbolic orbits and thus the outgoing energy fields from the event horizon can be found more precisely; allowing for the adequate signal processing analysis to be found for such a field.
\end{abstract}

Keywords: Stationary black holes; General relativity; Fourier analysis; Hyperbolic orbits; Quasi-gravity; Geometric scattering

\section{Résumé}

Un trou noir avec un moment cinétique nul est dit stationnaire. Dans certaines conditions, un tel trou noir peut être représenté comme une sphère. Cette article examine le théorème de topologie de Hawking, la métrique de Schwarzschild, les nouvelles solutions aux équations d'Einstein, les résonances des orbites hyperboliques autour de l'horizon des événements pour les trous noirs stationnaires et sphériques, et analyse leur importance. Dans le cas du trou noir sphérique stationnaire, on suggère l'utilisation de l'analyse de Fourier pour trouver les résonances dues à la dispersion géométrique des orbites hyperboliques. Ainsi, on peut trouver les champs énergétiques sortants de l'horizon des événements de façon plus précise ; permettant l'analyse de traitement du signal adéquat pour un tel champ.

Mots Clés: Trous noirs stationnaires; Relativité générale; Analyse de Fourier; Orbites hyperboliques; Quasi-gravité; Diffusion géométrique

\section{Introduction}

In 1915, on the front lines of World War I, Schwarzschild calculated and discovered, through Einstein's equations, large masses that significantly distort the fabric of spacetime that are known today as black holes. In this review, Hawking's topology theorem, and the special case of the Kerr metric of stationary black holes are examined. Furthermore, novel solutions to Einstein's equations, and resonances due to geometric scattering of hyperbolic orbits around the event horizon of stationary spherical black holes are outlined, and their hallmarks are discussed. This paper aims to offer an introductory outlook on the topology of spherical black holes. Moreover, it is argued that finding a spherical metric representing the hyperbolic behaviour of matter around the

Correspondence: bpuza021@uottawa.ca

Department of Physics, University of Ottawa, 598 King Edward, K1N 6N5,

Ottawa, Canada event horizons of stationary black holes will allow for adequate Fourier analysis calculations to obtain the photonic energy fields radiating away from the event horizon.

\section{Schwarzschild metric: a special case of the Kerr metric and null geodesics}

The metric that represents the stationary axisymmetric (that is, in the well-behaved outside domain rotating case when the Killing vector that generates the stationary symmetry becomes spacelike) black hole equilibrium state can be represented by the following Kerr metric:

$$
\begin{aligned}
d s^{2} & =\left(r^{2}+a^{2} \cos ^{2} \theta\right)\left(d \theta^{2}+\sin ^{2} \phi^{2}\right) \\
& +2\left(d u+a \sin ^{2} \theta d \phi\right)\left(d r+a \sin ^{2} \theta d \phi\right) \\
& -\left(1-\frac{2 m r}{r^{2}+a^{2} \cos ^{2} \theta}\right)\left(d u+a \sin ^{2} \theta d \phi\right)^{2}
\end{aligned}
$$


where $a$ is a real constant, $r$ is the radius from the event horizon, and $u$ is the time element (1). When the angular momentum $m a$ and the Schwarzschild mass $m$ are zero, then the special case of the Kerr metric, called the Schwarzschild metric, can be seen to be:

$$
\begin{aligned}
d s^{2} & =r^{2}\left(d \theta^{2}+\sin ^{2} \theta d \phi^{2}\right) \\
& +\frac{d r^{2}}{1-\frac{2 m}{r}} \\
& -\left(1-\frac{2 m}{r}\right) d t^{2}
\end{aligned}
$$

where $r=2 m$ is the location where the field becomes weak in terms of the mass (1).

When an outgoing null coordinate patch is analyzed, the Kerr metric at $a=0$ becomes:

$$
\begin{aligned}
d s^{2} & =-\left(1-\frac{2 m}{r}\right) d u^{2}+2 d u d r \\
& +r^{2} d u d r+r^{2} d \theta^{2} \\
& +\sin ^{2} \theta d \phi^{2}
\end{aligned}
$$

where $x^{0}=u, x^{1}=r, x^{2}=\theta, x^{3}=\phi(1)$, that is the usual spherical coordinate system.

\section{Hawking's Topology Theorem: The Stationary Case}

It has been shown that non-rotating stars of $M>M_{\odot}$ (solar mass) will eventually deplete all of their nuclear fuel and undertake catastrophic collapse, causing a significant distortion in the fabric of spacetime. However, if such a collapse is said to be spherically symmetric, then it can be characterized using the Schwarzschild metric (2). The Schwarzschild metric obeys the following characteristics:

1. The star's surface will exist inside the Schwarzschild radius $R=\frac{2 G}{c^{2}} M$, where $G$ is the universal gravitational constant, $c$ is the speed of light and $M$ is the mass of the black hole (2). Such a surface is said to be a closed trapped surface; that is, a space-like 2-surface such that both future directed families of null geodesics converge at each's respective future directed families of null geodesics orthogonal to it converge i.e. such a strong gravitational field that outwardly travelling light is pushed inwards will exist) $(3,4)$.

2. There exists a spacetime singularity (2).

3. An observer positioned outside the singularity and the Schwarzschild radius will not be able to physically observe the inside of the black hole (2). Therefore, physical theories inside the Schwarzschild radius are said to be unachievable (2). However, through the Cauchy data on the spacelike surface outside and near the Schwarzschild radius, physical theories can be predicted (2).
If such a collapse occurs under these conditions (5) and obeys the Schwarzschild metric, it must then be strongly asymptotic and thus stationary (2). Indeed, matter in proximity to this space obeys Maxwell's equations; therefore, the matter in this space can be described with wellbehaved hyperbolic equations (2). Evidently, asymptotic stationary black holes will have a Killing vector tangent to the event horizon because they are invariant under the horizontal parameter transformation (2). Moreover, Israel $(5,6)$ showed that if the black hole is stationary, static, and is empty or contains only a Maxwell field, then the solution will have spherical symmetry. However, if the space is not empty then the solutions on the event horizons will be directed along the null generators (2).

A general result of Lichnerowicz (7) conveys that within a stationary and empty black hole there exists a region such that the Killing vector is spacelike (2). One can conclude that the event horizon of this space will be rotating with respect to infinity because a particle that has a trajectory along the null geodesic generator of the horizon is moving with respect to the stationary frame (or the integral curves of the Killing vector) (2).

Essentially, Hawking's black hole topology theorem forms the event horizon into cross sectional spheres in the case where the black hole obeys the dominant energy condition (that is the condition that allows the massenergy of the universe can never flow at a speed faster then the speed of light and contributes to the evolution of universe) and is stationary in four dimensional asymptotically flat spacetimes $(2,8)$.

Galloway and Schoen (9) extended Hawking's theorem of black hole topology to higher dimensions and concluded that in higher dimensions, stationary black holes are not necessarily spherical (7). It was shown that the spacetime $\left(M^{\pi+1}, g\right)$ obeying the dominant energy condition also satisfies the Einstein equations:

$$
R_{a b}-\frac{1}{2} R g_{a b}=T_{a b}
$$

if, in addition, the energy-momentum tensor $T$ satisfies $T(X, Y)=T_{a b} X^{a} Y^{a} \geq 0$ for the vectors $\mathrm{X}, \mathrm{Y}(7)$. For dimensions $n \geq 3$, there exists a space-like hypersurface in the space-time $\left(M^{\pi+1}, g\right)$ ( 7$)$.If the outer apparent horizon, $\sum^{n-1}$ is of positive in $V^{n}$ unless $n=3,4$ (or Ricci flat) that is of induced metric and thus both the tensors $X$ and $T_{a b} U^{a} K^{b}$ vanish on $\sum(7)$.

A special solution that exists for dimension $\operatorname{dim} M=$ 5 or $\operatorname{dim} \sum=3$ (assuming $\sum$ is orientable) is due to calculations by Schoen-Yau $(10,11)$ and Gromov-Lawson $(12,13)$. The $\sum$ 's topology is a finite connected spherical sum of spaces (7). This sum of spherical spaces can be expressed as a connected sum of spheres by the prime decomposition theorem ( 7$)$. 
Hence, Hawking's topology theorem elegantly states that when black holes are stationary they can unquestionably be represented topologically as spheres. Such a topology can be manipulated mathematically to find the signals of the energy fields of photons nearby through Fourier analysis. The energy fields of photons will be discussed in the upcoming sections.

\section{Solutions to Einstein's Equations in Spherical Black Holes}

The theory of quasi-topological gravity has well-defined properties on spherically symmetric backgrounds (1419) that allow the field equations to reduce to second order differential equations with exact solutions (20). If one were to maximize these symmetric spherical backgrounds, then the corresponding linearized equations of motion would suitably coincide with the Einsteinian linearized equations (20). While quasi-gravity is a useful theory for its avoiding the extra degrees of freedom required in Einsteinian gravity, it only becomes non-trivial in dimensions (20).

Where the theory of quasi-gravity is non-trivial in dimensions $n \geq 5$, the theory of Einsteinian cubic gravity is non-trivial in four dimensions, consequently allowing for static spherically symmetric solutions (21, 22) to be represented as:

$$
d s^{2}=-N^{2} f d t^{2}+\frac{d r^{2}}{f}+r^{2} d \sum_{(d-2) k}^{2}
$$

where $N$ is a constant, and $d \sum_{(d-2) k}^{2}$ is the line element on a surface of constant scalar curvature $K=+1,0,-1$ corresponding respectively to spherical, flat and hyperbolic topologies (20). The solution (4) to the Einsteinian cubic gravity in four dimensions has a Schwarzschild-like solution that is not true in higher dimensions (21,22).

The general metric for all dimensions was discovered by Hennigar et al. [20]:

$$
f(r)=4 \pi T\left(r-r_{+}\right)+\sum_{i=2}^{n} a_{n}\left(r-r_{+}\right)^{n}
$$

where $T$ is the Hawking temperature, $r$ is the radius of the event horizon, $r_{+}$is the outward radius from the black hole, and $a_{n}$ is the usual Taylor Series expansion coefficient (20).

The Taylor series expansion about $f(r)$ shows the difference between the black hole's outer radius $r_{+}$and its inner radius $r$. This difference characterizes the behaviour near the event horizon (20). Therefore, Hennigar et al.'s quasi-topological gravity describes gravitational fields around the event horizon of black holes and compares the numerical results as the fields are emitted. The expansion about the event horizon of a spherical and the asymptotical expansion of a flat black hole are consistent; hence, the theory was seen to be numerically accurate.

Nevertheless, Hennigar et al. do not assess what are the physical implication of their quasi-topological gravity theory.

\section{Geometric Scattering and Resonances Around the Event Horizon}

Geometric scattering is a popular model (23-26) that is used when describing the association between the resonances and a representative sphere of hyperbolic orbits in the stationary black hole case. Quasi-normal modes (resonances) (27) on a well-defined strip below the real axis with angular momenta $l$ can be approximated by the "pseudo-poles" (28):

$$
\left( \pm 1 \pm \frac{1}{2}-\frac{i}{2}\left(k+\frac{1}{2}\right)\right) \frac{\left(1-9 \Lambda m^{2}\right)^{1 / 2}}{3^{3 / 2} m}
$$

where $l=1,2, \ldots, k(k=0,1, \ldots)$ with the resonances having multiplicity $2 l+1, \Lambda$ is the cosmological constant, and $m$ is the mass of the black hole. Recall that the Schwarzschild stationary black hole case occurs when $\Lambda=0$ (more specifically, when the angular momentum is zero).

The resonances of black holes can be thought of as pure tones, or more precisely; as frequencies and rates of damping of signals emitted by the black hole while in the company of perturbations (27). Therefore, the real part of a black hole resonance relates to the frequency of the emitted black hole signals while the imaginary part of the resonance relates to the signals' rate of decay in time (28). These significantly large pure tone resonance times are a property of the black hole and thus become independent of the perturbation (28). On the other hand, the stability of such a system under the perturbation will correspond to the distance from a reference point of the resonances (on the real axis) and thus at significantly larger distances the system will be more stable (28).

A hyperbolic operator to Schwarzschild black hole for the exterior of a black hole is:

$$
\boldsymbol{\square} g=\alpha^{-2} D_{t}^{2}-\alpha^{2} r^{-2} D_{r}\left(r^{2} \alpha^{2}\right)-D_{r} \alpha^{2} r^{-2} \Delta_{w}
$$

where $\alpha=\left(1-\frac{2 m}{r}-\frac{1}{3} \Lambda r^{2}\right)^{1 / 2}$, and $D=\frac{1}{i} \partial$ and $\Delta_{w}$ are the positive Laplacian on the 2-surface (28-30). Correspondingly, particular resonances in the stationary scattering phenomena with special solutions of (8) $\boldsymbol{\square}_{g} u=0$, relating to the following operator on $X$ :

$$
P=\alpha^{-2} r^{-2} D_{r}\left(r^{2} \alpha^{2}\right) D_{r}+\alpha^{2} r^{-2} \Delta_{w}
$$

where $P$ is the scattering resonance operator (28). Through the method of the separation of variables and 
the Regge-Wheeler transformation, a family of onedimensional Schrödinger operators with potentials decaying at infinity that has some unique non-degenerate maxima can be acquired (30). This resulting family of one-dimensional Schrödinger operators can be represented by the Hamiltonian that is required to constitute a single hyperbolic trajectory that unfolds the trapped set from the classical flow of the black hole (28).

Evidently, the pure tones of black holes can be found, generally, by the Fourier analysis for hyperbolic orbits of spherical black holes. This will allow for a more rigorously general theorem of energy fields of particles being outward emitted by the stationary black holes then the manipulation of the spherical symmetry technique.

\section{Conclusion}

Hawking's topology theorem, and the special case of the Kerr metric of stationary black holes are examined. Furthermore, novel solutions to Einstein's equations, and resonances due to geometric scattering of hyperbolic orbits around the event horizon of stationary black holes are outlined and their hallmarks are discussed. On nonEuclidean space-times such as the extremely distorted Hawking topological space-time present around black holes, there do not exist theorems for the resonances on hyperbolic trajectories. Therefore, the spherical symmetry of the black hole must be manipulated order to find the resonances. Consequently, this paper suggests performing an appropriate volume transformation with a spherical Laplacian on the Schwarzschild metric such that it is then transformed into Fourier space. As a result, a theorem that describes the resonances of the hyperbolic trajectories can be found that is more exact and general then the spherical symmetry approach. In return, by applying the Fourier analysis in the spherical black hole case, a more exact theorem of the frequency that is emitted from the spherical black hole's signals under perturbations will be found. Indeed, future research using Fourier analysis will allow for the resulting frequency and decay in signal time to be articulated into a more precise theorem that describes energy fields emitted outwardly from the event horizon to infinity through signal processing in extremely distorted space-times with Hawking's topology-like geodesics.

\section{Competing interests}

The author declares that he has no competing interests.

References

1. B. Carter, AIP Conference Proceedings 841, 29 (2006).

2. S. W. Hawking, Comm. Math. Phys. 25, 152 (1972).

3. R. Penrose, Phys. Rev. Lett. 14, 57 (1965).

4. C. DeWitt-Morette, J. Wheeler, B. S. R. Center, B. M. Institute, Battelle Rencontres: 1967 Lectures in Mathematics and Physics (Benjamin, 1968)

5. W. Israel, Phys. Rev. 164, 1776 (1967)

6. W. Israel, Comm. Math. Phys. 8, 245 (1968).
7. A. Lichnerowicz, C. R. Acad. Sci. Paris Ser. A.

8. S. Hawking, G. Ellis, The Large Scale Structure of Space-Time, Cambridge Monographs on Mathem (Cambridge University Press, 1973).

9. G. J. Galloway, R. Schoen, Commun. Math. Phys. 266, 571 (2006).

10. R. Schoen, S.-T. Yau, Annals of Mathematics 110, 127 (1979).

11. R. Schoen, S. T. Yau, manuscripta mathematica 28, 159 (1979).

12. M. Gromov, H. B. Lawson, Annals of Mathematics 111, 209 (1980).

13. M. Gromov, H. Lawson, Publications Mathematiques de l'Institut des Hautes Etudes Scientifiques 58, 83 (1983).

14. J. Oliva, S. Ray, Classical and Quantum Gravity 27, 225002 (2010).

15. J. Oliva, S. Ray, Phys. Rev. D 82, 124030 (2010).

16. R. C. Myers, B. Robinson, Journal of High Energy Physics 2010, 67 (2010).

17. R. C. Myers, M. F. Paulos, A. Sinha, Journal of High Energy Physics 2010, 35 (2010).

18. A. Cisterna, L. Guajardo, M. Hassaïne, J. Oliva, Journal of High Energy Physics 2017, 66 (2017).

19. A. Ghodsi, F. Najafi, The European Physical Journal C 77, 559 (2017).

20. R. A. Hennigar, D. Kubizňák, R. B. Mann, Phys. Rev. D 95, 104042 (2017).

21. R. A. Hennigar, R. B. Mann, Phys. Rev. D 95, 064055 (2017).

22. P. Bueno, P. A. Cano, Phys. Rev. D 94, 124051 (2016).

23. P. Briet, J. Combes, P. Duclos, Journal of Mathematical Analysis and Applications 126, 90 (1987).

24. C. Gérard, Mémoires de la Société Mathématique de France 31, 1 (1988).

25. R. R. Mazzeo, R. B. Melrose, Journal of Functional Analysis 75, 260 (1987).

26. M. Zworski, Inventiones mathematicae 136, 353 (1999).

27. S. Chandrasekhar, The Mathematical Theory of Black Holes (Springer Netherlands, Dordrecht, 1984), pp. 5-26.

28. A. S. Barreto, M. Zworski, Math. Res. Lett. 4, 103 (1997).

29. S. Chandrasekhar, S. Detweiler, Proceedings of the Royal Society of London. Series A, Mathematical and Physical Sciences 344, 441 (1975).

30. M.-B. A. Bachelot, Alain, Annales de l'I.H.P. Physique théorique 59, 3 (1993). 